\title{
Дискретная дифракция в массиве волноводов и оптические плоские зоны
}

\author{
А.И. Маймистов \\ Национальный исследовательский ядерный университет МИФИ, Москва \\ *E-mail: aimaimistov@gmail.com
}

DOI:10.31868/RFL2018.30-33

Давно привлекает внимание исследователей ситуация, когда электромагнитное излучение локализовано в волноводах, образующих одномерный или двумерный массив, и может проникать только ближайшие соседние волноводы за счет нарушения полного внутреннего отражения. Это напоминает систему электронов в кристалле, когда в условиях сильной связи электрон может перемещаться между ближайшими атомами решетки только за счет туннелирования.

Одномерная цепочка одинаковых волноводов была исследована в [1]. Было найдено, что если излучение интенсивностью $I_{0}$ вводится в центральный волновод, помеченный индексом 0 , то для интенсивности излучения в волноводе с номером $n$ на длине волновода $L$ было найдено выражение $I_{n}(L)=I_{0} J_{n}^{2}(2 K L)$, где $K$ - коэффициент связи соседних волноводов и $J_{n}(z)$ - функция Бесселя $n$-того порядка. Точно такое же выражение описывает поле при дифракции в режим Рамана-Ната (дифракция на тонкой решетке). Эта аналогия позволяет говорить об образовании распределения полей по волноводам цепочки как о дискретной дифракции.

В недавнее время появились работы, посвященные исследованию дискретной дифракции в более сложных цепочках волноводов [2-5]. Здесь будет рассматриваться бинарная (двухкомпонентная) цепочка волноводов, которая образована либо из волноводов с различными показателями преломления, либо из одинаковых волноводов, но с чередованием расстояния между соседними волноводами, либо из волноводов с положительным показателем преломления, но имеющих некоторое различие (показатель преломления или толщина волновода), как показано на Рис.1(а). Элементарная ячейка содержит два типа узлов - А и В.

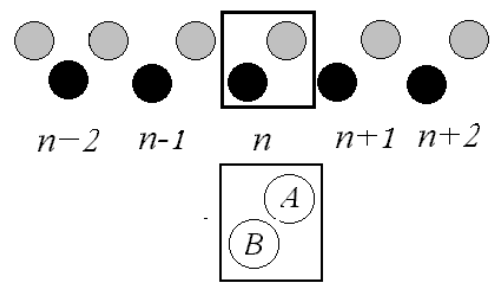

(a)

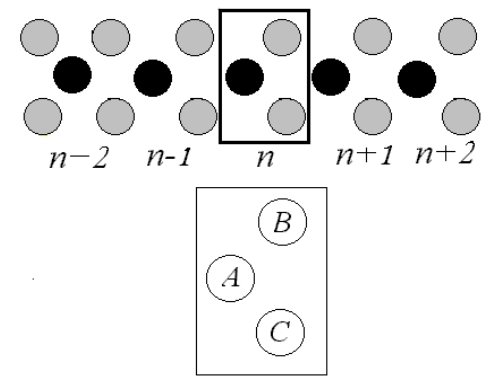

(б)

Рис. 1. Конфигурация бинарной цепочки (a) и ромбической цепочки. Прямоугольником указана элементарная ячейка.

В приближении медленно меняющихся амплитуд изменения напряженности электрических полей непрерывного электромагнитного излучения в волноводе каждого типа, определяются системой уравнений,

$$
i \partial_{\zeta} A_{n}=B_{n}+B_{n-1}, \quad i \partial_{\zeta} B_{n}=A_{n}+A_{n+1},
$$


где $A_{n}$ и $B_{n}$ - нормированные амплитуды электрических полей в волноводе $n$ той элементарной ячейки, безразмерная координата $\zeta$ есть пройденное волной расстояние вдоль оси волновода, измеренное в единицах длины связи [1]. Решение уравнений (1) можно получить различными способами, например, используя метод производящих функций. Общее решение зависит от начальных условий $A_{n}(0)$ и $B_{n}(0)$, выбрав которые можно записать $A_{n}(\zeta)$ и $B_{n}(\zeta)$ для любого значения $\zeta$. Наиболее простой вариант начальных условий таков: $A_{n}(0)=A_{0} \delta_{n 0}$ и $B_{n}(0)=0$. В этом случае распределение напряженностей полей по волноводам имеет вид

$$
A_{n}(\zeta)=A_{0}(-1)^{n} J_{2 n}(2 \zeta), \quad B_{n}(\zeta)=-i A_{0}(-1)^{n} J_{2 n+1}(2 \zeta),
$$

Это распределение напряженностей полей описывает дискретную дифракцию в бинарной цепочке волноводов. В предельном случае одинаковых волноводов эти выражения переходят в формулы, полученные в [1].

Если положить начальные условия такие, что излучение вводится в волноводы только одной (центральной, например) элементарной ячейки с номерм $n=0$, то есть $A_{n}(0)=A_{0} \delta_{n 0}$ и $B_{n}(0)=B_{0} \delta_{n 0}$, то решение уравнений (1) представляет распределения полей по волноводам, которые будут иметь следующий вид

$$
\begin{aligned}
& A_{n}(\zeta)=A_{0} J_{0}(2 \zeta) \delta_{n 0}+(-1)^{n} A_{0} J_{2 n}(2 \zeta)+i(-1)^{n} B_{0} J_{2 n-1}(2 \zeta), \\
& B_{n}(\zeta)=B_{0} J_{0}(2 \zeta) \delta_{n 0}+(-1)^{n} B_{0} J_{2 n}(2 \zeta)+i(-1)^{n} A_{0} J_{2 n+1}(2 \zeta),
\end{aligned}
$$

где $n= \pm 1, \pm 2, \ldots$. Третье слагаемое в этих выражениях описывает интерференцию полей в волноводах. Для более сложных случаев начальных условий, выражения для электрических полей в волноводах содержат слагаемые, учитывающие интерференцию полей из различных, не только соседних волноводов. Надо заметить, что если начальные условия выбраны в виде $A_{n}(0)=(-1)^{n} A_{0}$ и $B_{n}(0)=(-1)^{n} B_{0}$, то дифракция отсутствует. Причина в том, что поля, проникающие в каждый волновод из ближайших соседей, имеют противоположные фазы и их интерфенция дает нулевой результат.

Если в цепочке, показанной на Рис. 1(a) дополнительно учесть взаимодействие между ближайшими однотипными волноводами, (то есть, следующими за ближайшими соседями), то получится модель зигзагообразного массива волноводов из [6,7]. Система уравнений, описывающая поля в такой системе волноводов, имеют вид

$$
i \partial_{\zeta} A_{n}=B_{n}+B_{n-1}+\gamma\left(A_{n-1}+A_{n+1}\right), \quad i \partial_{\zeta} B_{n}=A_{n}+A_{n+1}+\gamma\left(B_{n-1}+B_{n+1}\right),
$$

где $\gamma$ есть отношение константы связи между следующими за ближайшими соседями и константы связи между ближайшими соседями в цепочке.

Решение этой системы уравнений может быть получено тем же методом производящих функций. Выбор конкретных начальных условий позволяет определить распределение амплитуд полей по волноводам $A_{n}(\zeta)$ и $B_{n}(\zeta)$ в глубине зигзагообразного массива волноводов.

Если рядом с зигзагообразной цепочкой расположить достаточно близко линейную цепочку волноводов, то получится ромбическая конфигурация 
одномерного массива волноводов, показанного на Рис. 1(б). Система уравнений, описывающая поля в таком массиве волноводов имеют вид

$$
i \partial_{\zeta} B_{n}=A_{n}+A_{n+1}, \quad i \partial_{\zeta} C_{n}=\gamma\left(A_{n}+A_{n+1}\right), \quad i \partial_{\zeta} A_{n}=B_{n}+B_{n-1}+\gamma\left(C_{n}+C_{n-1}\right),
$$

В случае симметричной конфигурации отношение констант связи $\gamma=1$.

Периодичность расположения волноводов в цепочке или в массиве приводит к формированию зонной структуры допустимых частот распространяющихся вдоль $\zeta$ волн. Интересным свойством рассматриваемой модели является то, что спектр распространяющихся волн имеет три ветви или три зоны. Две из них отвечают волнам, распространяющимся по волноводам и проникающим в соседние волноводы, что дает дискретную дифракцию в массиве волноводов. Третья ветвь спектра отвечает волнам бегущим вдоль оси волноводов, но не проникающих в соседние волноводы. Ветвь дисперсионного соотношения, отвечающая недифрагирующим волнам, имеет нулевую кривизну и потому соответствующая ей зона называется плоской зоной, по аналогии с подобными зонами квазичастиц в твердых телах $[8,9]$.

Систему уравнений (3) можно решить точно [10]. Выражение для общего решения очень громоздкое. В частном случае, если положить начальные условия такие, что излучение вводится в волноводы только одной элементарной ячейки, но так $A_{n}(0)=0$ и $B_{n}(0)=-C_{n}(0)=B_{0} \delta_{n 0}$, из решения уравнений (3) следующий, что $A_{n}(\zeta)=0$ и $B_{n}(\zeta)=-C_{n}(\zeta)=B_{0} \delta_{n 0}$. То есть, для такого выбора начальных условий дифракция отсутствует. Однако, если в один из волноводов типа А водится излучение, то есть если начальными условиями будут $A_{n}(0)=A_{0} \delta_{0 n}$ и $B_{n}(0)=-C_{n}(0)=B_{0} \delta_{n 0}$, то результирующее распределение полей будет следующим

$$
\begin{gathered}
A_{n}(\zeta)=(-1)^{n} A_{0} J_{2 n}(\sqrt{2} \zeta) \\
B_{n}(\zeta)=B_{0} \delta_{n 0}-(-1)^{n} 2^{1 / 2} i A_{0} J_{2 n+1}(\sqrt{2} \zeta), \quad C_{n}(\zeta)=-B_{0} \delta_{n 0}-(-1)^{n} 2^{1 / 2} i A_{0} J_{2 n+1}(\sqrt{2} \zeta) .
\end{gathered}
$$

Так что дискретная дифракция имеет место. Любое отклонение от начального условия, отвечающего возбуждению мод плоской зоны, влечет расплывание первоначально локализованного возбуждения.

Иногда нелинейные свойства среды, в которой распространяются электромагнитные волны, приводят к локализации волны. Дисперсия или дифракция оказываются подавленными и формируются уединенные волны. Потому имеет смысл рассмотреть обобщения рассмотренных моделей. Учет нелинейных свойств волноводов ромбической решетки выполнен в предположении, что нелинейность кубическая. Система уравнений, обобщающая (3), имеет следующий вид

$$
\begin{aligned}
i \partial_{\zeta} B_{n}= & A_{n}+A_{n+1}+\mu\left|B_{n}\right|^{2} B_{b}, \quad i \partial_{\zeta} C_{n}=\gamma\left(A_{n}+A_{n+1}\right)+\mu\left|C_{n}\right|^{2} C_{b}, \\
& i \partial_{\zeta} A_{n}=B_{n}+B_{n-1}+\gamma\left(C_{n}+C_{n-1}\right)+\mu\left|A_{n}\right|^{2} A_{b},
\end{aligned}
$$

Здесь характеризующий нелинейность параметр $\mu$ выбран одним и тем же для всех волноводов. Далее рассматривается случай симметричной решетки, когда $\gamma=1$.

Из системы уравнений (4) следует выражение, которое связывает плотности «энергии»

$$
W_{n}=\left|A_{n}\right|^{2}+\left|B_{n}\right|^{2}+\left|C_{n}\right|^{2}
$$
«ТОК» 
$f_{n}=i A_{n}\left(B_{n-1}^{*}+C_{n-1}^{*}\right)-i A_{n}^{*}\left(B_{n-1}+C_{n-1}\right) \quad$ и $\quad$ которое имеет вид уравнения непрерывности: $\partial_{\zeta} W_{n}+\left(f_{n+1}-f_{n}\right)=0$. В двух случаях

(a) $A_{n}=0$ и $B_{n}=-C_{n} ; \quad$ (б) $A_{n}=(-1)^{n} A, B_{n}=(-1)^{n} B$ и $C_{n}=(-1)^{n} C$,

ток равен нулю, что означает отсутствие дифракции в ромбической решетке нелинейных волноводов.

Можно показать, что существует решение системы уравнений (4), которое удовлетворяет первому условию. Это решение дает следующее распределение амплитуд полей

$$
A_{n}(\zeta)=0, \quad B_{n}(\zeta)=-C_{n}(\zeta)=b_{0} \exp \left(-i \mu b_{0}^{2} \zeta\right) .
$$

Решение, отвечающее второму условию (то есть, условию (б)) имеет вид распределения полей

$$
\begin{gathered}
A_{n}(\zeta)=(-1)^{n} a_{0} \exp \left(-i \mu a_{0}^{2} \zeta\right), B_{n}(\zeta)=(-1)^{n} b_{0} \exp \left(-i \mu a_{0}^{2} \zeta\right), \\
C_{n}(\zeta)=(-1)^{n} c_{0} \exp \left(-i \mu a_{0}^{2} \zeta\right)
\end{gathered}
$$

В этих выражениях вещественные параметры $b_{0}$ и $c_{0}$ связаны соотношением $b_{0}^{2}=c_{0}^{2}$, а параметр $a_{0}$ произвольный.

Распределение (5) неустойчиво относительно малых возмущений амплитуд в волноводах, до тех пор, пока величина $\mu b_{0}^{2}$ не превысит определенное пороговое значение. После этого малые возмущения не затухают, но и не растут экспоненциально [11]. Напротив, распределение, отвечающее случаю (б) устойчиво до тех пор, пока величина $\mu a_{0}^{2}$ не достигнет порогового значения, после чего развивается модуляционная неустойчивость. Таким образом, моды плоской зоны неустойчивы. В нелинейном случае превышение некоторой пороговой интенсивности вводимого в ромбическую решетку волноводов излучения может стабилизировать распределение полей по волноводам. Пока не ясно, возможно ли образование «дискретный солитон» в этом случае.

Мне доставляет удовольствие поблагодарить А.С. Десятникова, В.А. Патрикеева и Е.И. Ляшко за полезные дискуссии и помощь в работе. Работа поддерживалась РФФИ (грант №. 18-02-00278).

\section{Литература}

[1] S.Somekh et al., Appl. Phys. Letts. 22, 46-47 (1973).

[2] S. Flach et al., Europhys. Lett. 105, 30001 (2014).

[3] S. Mukherjee et al., Opt. Lett. 40, 5443-5446 (2015).

[4] M. Nita et al., Phys.Rev. B. 87, 125428 (2013).

[5] L. Morales-Inostroza and R. A. Vicencio, Phys. Rev. A. 94, 043831 (2016).

[6] N.K. Efremidis and D. N. Christodoulides, Phys. Rev. B. 65, 056607 (2002).

[7] Е.В. Казанцева, А.И. Маймистов, Квантовая электрон. 43, 807-813 (2013).

[8] V. Apaja, M. Hyrkäs and M. Manninen, Phys.Rev. B. 82, 041402(R) (2010).

[9] C. Weeks and M. Franz, Phys. Rev E. 82, 085310 (2010).

[10] A.I. Maimistov and V.A. Patrikeev, J.Phys. Conf.Series 737, 012008 (2016).

[11] A.I. Maimistov, J. Opt. 19, 045502 (2017). 